\title{
CLIMATE SENSITIVITY AND EQUILIBRIUM CLIMATE STATES OF A TWO-DIMENSIONAL ENERGY BALANCE MODEL
}

\author{
(Abstract) \\ by \\ Jin Huang and Kenneth P. Bowman \\ (Department of Atmospheric Sciences, University of Illinois, \\ 105 S. Gregory Avenue, Urbana, IL 61801, U.S.A.)
}

A two-dimensional energy balance model (EBM) with a seasonal cycle and a realistic land-ocean distribution is used to study climate sensitivity and the properties of equilibrium climate states. The land-ocean distribution is represented by the heat capacity of the surface. The horizontal heat flux is parameterized by diffusion. Nonlinearity is introduced into the model by the albedo-temperature feedback. A multi-grid finite difference method is used to solve the model equation. This method shows great advantages compared to other numerical methods.

The sensitivity of snow cover to changes in the solar constant is examined. It is found that the climate is more sensitive in summer due to a more effective albedotemperature feedback. The local annual mean temperature and the amplitude of the seasonal cycle depend on the land-ocean distribution. Because Eurasia has the largest annual cycle of temperature and is the coldest in winter, winter snow appears first in Eurasia as the solar constant is reduced. Wintertime snow appears next in Greenland because of its low annual mean temperature and last in North America. Perennial snow cover appears first in Greenland because of the low annual mean temperature and the small annual cycle, and next appears in North America. Perennial snow in Eurasia appears only when the solar constant is reduced to a very low value, since the Eurasian land mass is too hot in summer for perennial snow cover. The sensitivity of the model climate to changes in the orbital parameters is also investigated and discussed.

Small polar ice caps in one-dimensional mean-annual EBMs are unstable to small perturbations. The ice caps either vanish or grow to a stable finite size. This phenomenon, referred to as the small ice-cap instability (SICI), is a consequence of the multiple stable equilibrium states in one-dimensional models. The SICI may be related to glacial-interglacial transitions. However, numerical results with the two-dimensional EBM demonstrate that the existence of the SICI depends on the land-ocean distribution. The SICI exists in the southern hemisphere, but not in the northern hemisphere. This casts doubt on the role of the SICI in northern hemisphere glaciations. Experiments with a one-dimensional seasonal EBM with simplified geography have been made to analyze the two-dimensional results. Mechanisms for the existence of the SICI in seasonal EBMs are discussed.

\section{THE EFFECT OF RHEOLOGY ON SEASONAL SEA-ICE SIMULATIONS}

\section{(Abstract)}

Bill Ip, W.D. Hibler, III, and Greg Flato

(Thayer School of Engineering, Dartmouth College, Hanover, NH 03755, U.S.A.)

On the seasonal time scales relevant to numerical investigations of climate, the rheology used in large-scale sea ice models significantly affects the ice thickness build-up and ice velocity fields. Plastic rheologies with a normal flow rule have been used to-date in seasonal dynamic thermodynamic simulations. These rheologies have proved useful in simulating discontinuous slip near the coast while still supplying relatively robust velocity fields in the central Arctic Basin. However, as indicated by limited numerical sensitivity studies with different types of elliptical yield curves, the amount of shear strength significantly affects the ice build-up and can possibly cause a stoppage of the ice outflow through Fram Strait. In addition to the shear strength issue, there is also the possibility that nonnormal flow rule rheologies, such as the Mohr Coulomb failure criterion used in soil mechanics, may cause somewhat different types of flow patterns, especially in the Fram Strait region. However, to date no seasonal simulations with such non-normal flow rule rheologies have been carried out.

In order to investigate the role of different rheologies on the large-scale flow patterns in the Arctic Basin, a more general numerical scheme than that of Hibler (1979) is developed, which allows the simulation of the dynamic thermodynamic behavior of sea ice with a wide variety of different non linear rheologies. Using this numerical scheme, comparative simulations are carried out to seasonal equilibrium with several variations of the Mohr Coulomb rheology and compared to the more standard Elliptical yield curve results. In particular, the main control Mohr Coulomb case is a capped rheology in which the shear strength is taken to be proportional to the compressive stress. In this capped case only shear flow is allowed until a maximum allowable compressive stress is reached. This cap strength is parameterized to be a function of the ice thickness and compactness. For comparison, a simulation with a very large cap strength is also carried out, and an experiment with a similar compressive cap but much lower shear strength. Overall the results are analyzed to determine the sensitivity of the ice build-up to flow rule and shear strength magnitude. In addition special attention is given to the character of the flow and stoppage (if any) through Fram Strait.

\section{REFERENCE}

Hibler, W.D., III. 1979. A dynamic thermodynamics sea ice model. J. Geophys. Res., 9, 815-846. 